\title{
Microbiological Analysis of Commercial Raw Meat-Based Diets for Dogs
}

\author{
Johnattan Castañeda Duque ${ }^{1, *}$, Luisa Becerra López ${ }^{1}$, Víctor M Molina $\mathrm{D}^{2}$, and Elkin Arboleda Z1 \\ ${ }^{1}$ Facultad de Ciencias Agrarias, Grupo de investigación Centauro, Universidad de Antioquia, Robledo-Medellín, Colombia \\ ${ }^{2}$ Boerhringer Ingelheim, Pets Technical Services, Bogotá, Colombia
}

*Corresponding author: Johnattan Castañeda Duque, Facultad de Ciencias Agrarias, grupo de investigación Centauro, Universidad de Antioquia, Robledo-Medellín-Colombia, E-mail: jonattan-2491@hotmail.com

Received: 08 Jun, 2020 | Accepted: 15 Jun, 2020 | Published: 26 Jun, 2020

Citation: Duque JC, López LB, Molina DVM, Arboleda ZE (2020) Microbiological Analysis of Commercial Raw Meat-Based Diets for Dogs. J Anim Sci Res 4(2): dx.doi.org/10.16966/2576-6457.140

Copyright: (C) 2020 Duque JC, et al. This is an open-access article distributed under the terms of the Creative Commons Attribution License, which permits unrestricted use, distribution, and reproduction in any medium, provided the original author and source are credited.

\section{Abstract}

Objective: Assess the sanitary quality of the commercial B.A.R.F diets to which they have access in the city Medellín (Colombia), performing microbiological evaluations through bacterial and fungal crops in a representative sample of diets based on raw meat for dogs.

Materials and Methods: 84 cultures were performed in 12 commercial RMDB or BARF diets, and were evaluated these parameters: growth of Salmonella spp, Clostridium spp, E. coli, total and fecal coliforms, aerobic mesophiles, fungi and yeasts.

Results: There was growth of the listed microorganisms: molds (50\%), yeasts (83\%), aerobic mesophiles (83\%), total coliforms (83\%), E coli (17\%), Clostridium perfringens (8\%), there was no Salmonella spp growth in any of the tested diets.

Conclusions: It was demonstrated the pathogen potential and possibly zoonotic of BARF diets, veterinarians and pet owners must know these results, in order to decide with real criterion if recommend or feed these diets to their pets.

Keywords: BARF; Microorganisms; Nutrition; Zoonoses

\section{Introduction}

In recent years, natural diets have gained great strength as a nutritional alternative for our pets $[1,2]$ and a dichotomy has been created between those who must be the type of food that should be given to dogs, natural foods affected in optimal food raw meat type BARF (raw appropriate organic food, ACBA Biologically Appropriate Raw Food) or RMBD (Raw meat-based diets; DBCC Diet based on raw meat), or commercial pellet-type foods, feeding is a crucial part of caring for keep patients healthy, however, many owners and even veterinary doctors have many doubts about these types of natural foods and do not know the benefits or problems to pets and public health that these foods can bring [3]. Today there are many commercial and homemade alternatives to feed dogs, among the many nutritional alternatives to offer to Pets. They are dry and wet foods in different presentations $[4,5]$.

The feeding of pets with RMBD or BARF diets, were affected in the 1980s by Australian veterinary doctor Ian Billinghurst, who has promoted this type of food, with writings on its apparent benefit $[4,5]$. These diets have become popular all over the world and have had a great boom in Europe, Asia and now in North America and Latin America, reaching Colombia and more than 5 years ago. It has become more than a way of feeding pets, for some, a lifestyle, looking for a diet based on the consumption of an ancestral diet, more natural $[1,6]$, however they do not take into account the thousands of years that have allowed the dog to differ from its ancestors by being domesticated, which through 36 changes in its genome regions, where 10 of these times can have a critical role in starch digestion and fat metabolism, that makes the feeding and even the life expectancy of dogs and wolves not the same [7].

B.A.R.F diets are foods that include a wide variety of ingredients, meat (chicken, pork, beef, fish, lamb), vegetables, fruits, eggs with or without shell, oils, dairy products, cereals, supplements, among others. All these ingredients are mixed by different processes and this allows raw meat diets to be offered in many commercial or homemade presentations $[1,3,7]$. Today this nutritional alternative in the city of Medellín, Colombia, has generated a large growing industry, where to date there are more than 30 companies that sell BARF diets. The components of these diets are prone to easy contamination with different bacterial and fungal agents that could have a pathogenic and toxic potential, not only for pets, but also for all people who handle these diets and are in contact with the animals that consume them $[2,4,5]$.

Proponents of BARF-type diets claim multiple benefits of these on the health of pets, such as strengthening the immune system, less presence of food allergies, canines and felines healthier, a longer life expectancy, better dental health, better coat and the skin $[4,7,8]$. 
However, all these benefits are anecdotal, and are not based on any type of study with scientific and statistical validity to support it, and it is based only on books published by the creator of the diet, which does not give real scientific validity to the benefits obtained, on the contrary in recent years, this topic has become relevant in public and veterinary health, and multiple studies have been conducted in countries such as the Netherlands, the Netherlands, Canada, USA, Germany, among others, where the pathogenic potential and the epidemiological importance that it could have on the health of pets and their owners when feeding them with this type of diet have been established [610]. Different authors have corroborated that contradictory to what the defenders of BARF diets say, this type of nutrition in pets can make them very prone to metabolic diseases, nutritional imbalances, dental fractures, intestinal and oesophageal perforations with bones, digestive infections, gastroenteritis, pancreatitis, allergic diseases and secondary nutritional hyperparathyroidism $[4,5,8,11]$.

Regarding public health, it is of particular interest to know that these foods not only have a great risk of having bacteria, any weight that can be eliminated almost entirely by different means of cooking and preparing commercial foods, in this case, no application. for BARF diets, since they must offer raw to dogs, which also allows a perfect breeding ground to be generated for exponential growth of CFU (Colony Forming Units) of bacterial and fungal agents that can also be endotoxin and mycotoxin releasers $[9,12]$. Therefore, the contact of animals and people with BARF-type diets represents a serious risk to public health and these microorganisms could be released into the environment by releasing said bacterial agents by contaminated animals or being directly in contact with humans who manipulate the BARF food [13].

Despite the studies that have already been carried out in different parts of the world, obtaining results that differ statistically from one to the other, with different percentages of bacterial growths in BARF diets [2,4-7], all leave ahead a alarming sign that these natural diets can cause harm to pets and humans $[1,2-7,9,11,14,15]$ however, in Colombia, no relevant study has been conducted on this topic and it is still unknown by owners and veterinary doctors the risks of these foods. For this reason, a microbiological study and identification of different bacterial and fungal agents were carried out in commercial BARF diets for canines in the city of Medellín-Colombia.

\section{Materials and Methods}

\section{Type of study}

The study carried out is descriptive, where there are discrete counting quantitative variables and binomial qualitative compliance variables.

\section{Study site}

The study was carried out in the city of Medellín, Colombia. Being the most populous city of the department according to the statistics of the National Administrative Department of Statistics-DANE. It is located in the widest part of the natural region known as Valle de Aburrá, with an altitude of 1,579 meters above sea level, an average temperature of $24^{\circ} \mathrm{C}$, geographic coordinates of latitude: 6,217, longitude: $-75,5676^{\circ} 13^{\prime} 1^{\prime \prime}$ North, $75^{\circ} 34^{\prime} 1^{\prime \prime}$ West.

\section{Selection of samples}

It began with an analysis of the current market for B.A.R.F type diets in the city of Medellín-Colombia, field work was carried out during 2018, investigating and learning about the different commercial brands of R.M.B.D food for canines.
In total, more than 30 commercial companies were found that manufacture and distribute their diets in different presentations, such as: food in Nuggets, sausage-type foods, foods with their liquefied components, diets with their slightly homogenized components in which it is possible to differentiate at a glance the ingredients and foods wrapped in bijao leaves (Calathea lutea A) and cabochon.

Of the companies mentioned, 10 companies were chosen randomly for convenience to carry out the analyzes. Some produce food from different animal origins as a source of protein, from which different types of food were taken. In total, 12 diets of 10 companies were analyzed, for a $n=12$ in the statistical analysis, using the formula proposed by Lwanga and Lemeshow (1991) for finite populations, to which 7 microbiological analyzes were performed individually, for a total of 84 total analyzes carried out on BARF diets Reliability was calculated with the formula reported by Cronbach (1943) and Cohen (2006), obtaining a reliability of $92 \%$. As an inclusion factor, all the diets that had an I.C.A (Colombian Agricultural Institute) register (4 diets) were taken.

\section{Analytical methods}

The researchers decide to carry out the analyzes through an external company, in order to reduce biases and have reliable data. The Test Laboratorio $^{\circ}$ food and water laboratory was used as a reference laboratory. Which was chosen for having ICA registration (Colombian Agricultural Institute) and also having the authorization by the Health Section of Medellín, for analysis of food and beverages? Taking the ICA regulations DIP-30-100-003, based on ICA resolutions No. 1056 of 1996 and 444 of 1993, as reference values of permissibility for bacterial growth.

Purchase, storage and distribution of diets. The 12 BARF diets were purchased the same day (April 24, 2018), directly from the production companies and were analyzed before the expiration date according to the labels in the cases of the products that had them, transportation was guaranteed with the freezing or refrigeration conditions according to the recommendations of the label until their analysis according to whether they had labels or not, until the analysis laboratory, which was carried out the same day of the purchase of the BARF diets.

\section{Laboratory methods}

The microbiological analyzes were carried out by Test Laboratorio ${ }^{\circ}$, which used its internal protocols for food processing. All the BARF type food delivered was received, labelled and identified to make the entire process traceable. The diets are allowed to thaw at room temperature, as would be done under normal conditions by the owner, to immediately offer them to the pet. Once the food is thawed or leaves refrigeration, the following cultures are started for each of them: samples of $25 \mathrm{~g}$ obtained from different parts of the diet were taken to obtain a representative sample of the matrix to be analyzed, these $25 \mathrm{~g}$ they were homogenized and subsequently subjected to different processes to identify the microorganisms.

The pathogens evaluated were: Salmonella spp, Clostridium spp, Escherichia coli, total and fecal coliforms, aerobic mesophiles, fungi and yeasts.

\section{Microbiological analysis}

The food analyzes were performed according to the internal protocols of the reference laboratory, and the INVIMA Ed. 1998 technique traceable to AOAC Official Methods 967.27 edition 17.

For the plate count of E. coli and coliforms, APHA 2001 CHAPTER 8 METHOD: 8,933 and NTC 4458 were taken into account. 
Protocols for microbiological cultures were carried out as can be seen in figure 1 .

A microbiological analysis was carried out, which established as parameters to be analyzed those that the ICA regulations establish as such to guarantee the microbiological safety of $\operatorname{dog}$ food.

Within these parameters, it is established (according to the DIP-30-100-003 page 2 of 3), Escherichia coli, count of mesophilic microorganisms, coliform microorganisms (total and faecal), Clostridium sulfite reducers, Salmonella spp, count of fungi and yeasts. Through this ICA directive, the allowance values of CFU/g (Colony Forming Units per gram) are established, for each microorganism, since pet food must be safe, but not sterile, and to a certain extent it is allows the presence of some microorganisms. However, in the cases of Salmonella spp and E. coli the permissibility is null, due to its high pathogenic and zoonotic risk (Table 1).

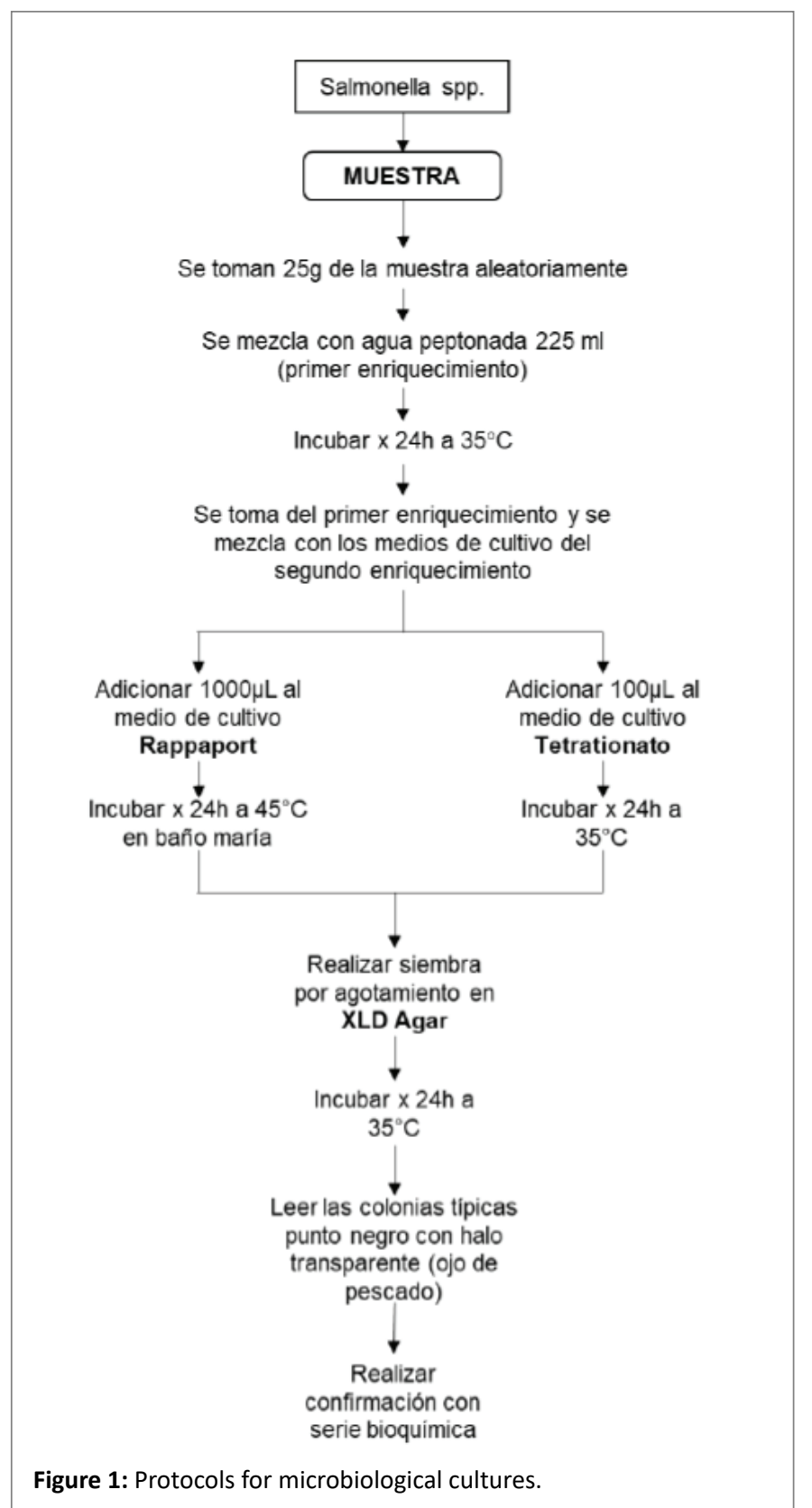

The results were analyzed based on global data and were subsequently related to the ICA entity's permissibility values established in 1997, which are still in force in Colombia.

A microbiological analysis was performed on the 12 BARF or RMBD type diets based on raw meat, which were subjected to these microbiological cultures for the following microorganisms: E. coli, Samonella spp, Total coliforms, Fecal coliforms, Clostridium perfringens, aerobic mesophilic microorganisms, molds and yeasts, for a total of 84 analyzes of the BARF-type diets evaluated.

\section{Statistic analysis}

It was performed using the $\mathrm{R}$ Studio statistical analysis software package, version 3.5.1, the sample calculation was performed using the formula proposed by Lwanga and Lemeshow (1991) for finite populations, and the reliability was found with the formula reported by Cronbach (1943) and Cohen (2006). The Chi square test was performed; in addition the relationship between the protein source and bacterial growth was analyzed.

\section{Ethical aspects}

This study did not involve live animals, only manipulation of raw BARF-type diets, which were obtained by the researchers directly from the manufacturers, their analyzes were not performed by them to avoid conflicts of interest and to be objective in the analyzes of the data and results obtained. The diets were delivered the same day of the acquisition to the TESTLAB ${ }^{\circledR}$ food and water analysis laboratory, and 15 days later the results were accessed.

During the study, the results and the companies analyzed were confidential and no brand was mentioned directly.

\section{Results}

Twelve raw meat diets were analyzed, which were obtained from 10 commercial companies that sell BARF or RMBD type diets in the city of Medellín-Colombia.1 diet per brand was analyzed. As can be seen in table 2 and figure 2. The labels on BARF-type diets revealed that $67 \%$ do not have an ICA registration (8 diets), a mandatory registration to market food in animals, as observed in figure $2.92 \%$ did not have all the information that would allow knowing the date of manufacture of

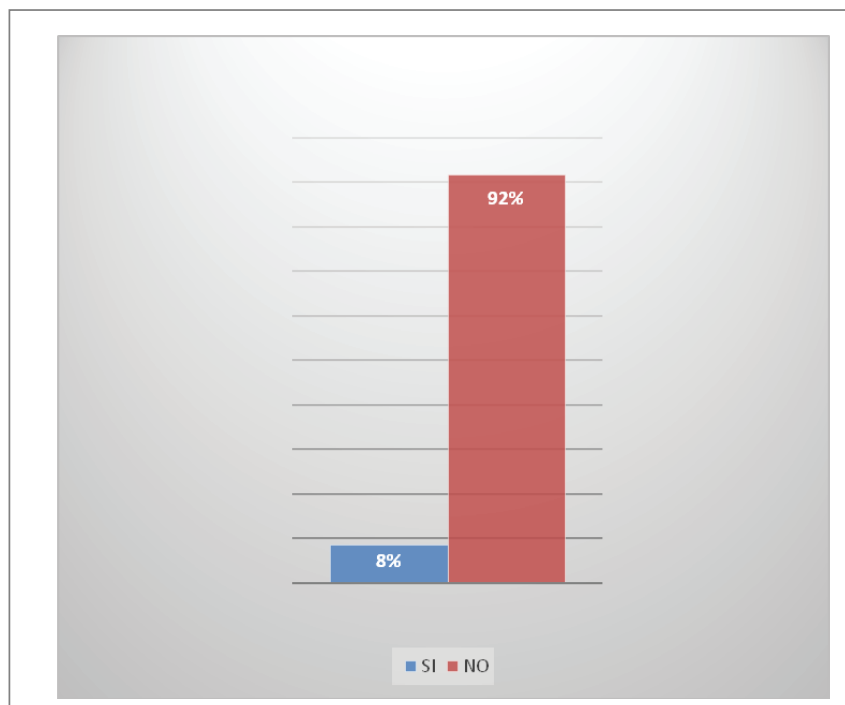

Figure 2: Compliance with ICA traceability and registration requirements. 
the diets ( 11 diets), $58 \%$ did not have information about the expiration date of the product ( 7 diets), $58 \%$ did not have a batch identification that allowed traceability of the product ( 7 diets) and $42 \%$ of the diets either did not have the ingredients in the label information or were not labeled ( 5 diets). Of the 12 diets analyzed, only 1 had all the mentioned parameters, considered essential to be able to adequately trace and ensure food safety.

The 12 diets were analyzed to identify the most used protein sources in BARF type raw meat diets, most of them did not have information about the protein source they used, as seen in figure 3 , among the sources that they were able to identify it was found that the most used was chicken with $25 \%$ ( 3 diets), followed by combinations of chicken and beef meat with $17 \%$ ( 2 diets), then turkey with $8 \%$ ( 1 diet) and beef with $8 \%$ ( 1 diet), a total of $42 \%$ had no information on their ingredients (5 diets).

In addition to protein sources, there were other ingredients such as fruits, vegetables, eggs, dairy, among others.

Relationship between the source of protein used and the growth of microorganisms. As can be seen in table 2. A greater growth of pathogenic microorganisms was found in the diets that had chicken as a single source of protein or combined with beef.

Table 3 lists the results with the variables it grows or it does not grow, assigning each company a letter, it is possible to show that in 39 of the 84 analyzes there was growth of microorganisms equivalent to $46 \%$.

The following growths were observed in each microbiological parameter: Molds grew in 6 of the 12 diets, for a total of $50 \%(p>0.05)$ and in the case of yeasts growth was observed in 10 of the 12 diets, for a $83 \%(p<0.05)$. Aerobic mesophilic growth was obtained in 10 of the 12 diets, for $83 \%(\mathrm{p}<0.05)$; Total coliforms grew in 10 of the 12 diets, for a total of $83 \%(p<0.05)$; there was no growth of Salmonella spp in any of the diets evaluated for $0 \%(\mathrm{p}<0.05)$, E. coli grew in 2 of the 12 diets ( $\mathrm{p}$ $<0.05$ ), for $17 \%$ in the count; Clostridium perfringens grew on 1 of the 12 diets, for $8 \%(\mathrm{p}<0.05)$ growth.

The permissible parameters of the ICA illustrated in table 3 were established, such as the reference values to know the sanitary quality of food for canines and establish their safety in the analyzed BARF diets. As can be seen in figure 3, in $75 \%$ of the diets analyzed there was growth of some bacteria, fungus or yeast above the values established as permissible.

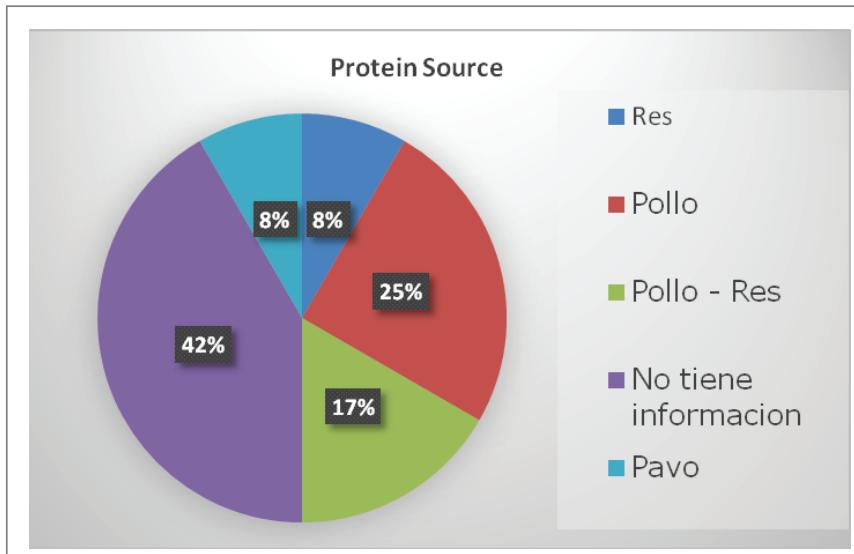

Figure 3: Protein sources used in the analyzed diets.
Table 1: Allowance for microbiological parameters in dog food, according to ICA 1997 (DIRECTIVE DIP-30-100-003 page 2 of 3).

\begin{tabular}{|c|c|}
\hline \multicolumn{2}{|c|}{ ESPECIE: CANINA } \\
\hline Parámetros Microbiológicos & UFC/g \\
\hline Mesophilic microorganism count. & $50 \times 10^{3}$ \\
\hline Total coliform microorganism count. & $10 \times 10^{2}$ \\
\hline Clostridium sulfite reducing count. & $10 \times 10^{1}$ \\
\hline I count yeast and fungi. & $10 \times 10^{2}$ \\
\hline Isolation Salmonella spp in 25g. & Ausente \\
\hline Isolation Escherichia coli. & Ausente \\
\hline
\end{tabular}

Table 2: Relationship between the source of protein used and the growth of microorganisms.

\begin{tabular}{|l|c|c|c|c|c|}
\hline & \multicolumn{5}{|c|}{$\%$ Protein Source } \\
\hline & Beef & Beef-Chicken & Turkey & Chicken & $\begin{array}{c}\text { It has no } \\
\text { information }\end{array}$ \\
\hline $\begin{array}{l}\text { No Bacteria, } \\
\text { Fungi, or Yeast } \\
\text { grew }\end{array}$ & $8 \%$ & $0 \%$ & $0 \%$ & $0 \%$ & $17 \%$ \\
\hline $\begin{array}{l}\text { Bacteria, Fungi } \\
\text { or Yeasts grew }\end{array}$ & $0 \%$ & $17 \%$ & $8 \%$ & $25 \%$ & $25 \%$ \\
\hline
\end{tabular}

Of the analyzed diets, all complied with the regulations regarding the growth of fungi and $100 \%$ of the BARF diets complied with the permissible levels in CFU/g despite the fact that in 6 of the diets fungi grew; $50 \%$ (6 diets) of the food grew yeasts above the $5000 \mathrm{CFU} / \mathrm{g}$ allowable by ICA regulations.

It is observed in table 3 . That $8 \%$ of the diets analyzed, that is, 1 diet had a growth of Clostridium perfringens of $9560 \mathrm{CFU} / \mathrm{g}$, a highly pathogenic microorganism of which the permissibility is less than 100 CFU/g in dog food. There was no growth of Salmonella in any of the 12 BARF-type diets evaluated where $100 \%$ complied with the regulations. Although there was growth of mesophilic microorganisms in 10 of the 12 diets analyzed, $100 \%$ of them had growths below $50,000 \mathrm{CFU} / \mathrm{g}$ complying with the ICA regulations. Of the analyzed diets $67 \%$ had total coliform growths greater than $1000 \mathrm{CFU} / \mathrm{g}$, that is, 8 diets did not meet the permissible levels and in the case of E. coli $17 \%$ of the analyzed diets had bacterial growths, being a bacterium that must be absent in canine diets according to ICA 1997 regulations.

\section{Discussion and Conclusion}

This study allowed us to recognize the current state regarding the sanitary and microbiological quality of the BARF-RMBD type diets in the city of Medellín-Colombia. Finding that $75 \%$ of the diets did not meet all the parameters established to consider the diets innocuous according to the ICA regulations, it is suggested to establish this type of food as highly dangerous for the health of the pets that consume it and people who eat it. Manipulate, which is consistent with other publications $[5,10,14]$.

In this study, no growths of Salmonella were found in the diets, which differs from other studies carried out in different parts of the world, where the findings of Salmonella in RMDB diets have ranged from 7 to $40 \%[2,4,5]$, where the PCR technique has been the most used analysis method, this would imply that our analysis method, which was bacteriological culture, is probably not the ideal one to look 
Table 3: Tabulation of bacterial growth in the analyzed diets.

\begin{tabular}{|c|c|c|c|c|c|c|c|}
\hline $\begin{array}{l}\text { Analysis/ } \\
\text { company }\end{array}$ & Mold Count & Yeast Count & $\begin{array}{c}\text { Clostridium } \\
\text { perfringens count }\end{array}$ & $\begin{array}{l}\text { Aerobic Mesophilic } \\
\text { Microorganism Count }\end{array}$ & $\begin{array}{c}\text { Total Coliform } \\
\text { Count }\end{array}$ & $\begin{array}{c}\text { Escherichia coli } \\
\text { count }\end{array}$ & $\begin{array}{c}\text { Detection of } \\
\text { Salmonella spp in } 25 \mathrm{~g}\end{array}$ \\
\hline A & Not grow & Not grow & Not grow & Not grow & Not grow & Not grow & Not grow \\
\hline B & Grow & Grow & Not grow & Grow & Grow & Not grow & Not grow \\
\hline C & Grow & Grow & Not grow & Grow & Grow & Not grow & Not grow \\
\hline D & Grow & Grow & Not grow & Grow & Grow & Not grow & Not grow \\
\hline E & Not grow & Grow & Not grow & Grow & Grow & Not grow & Not grow \\
\hline $\mathrm{F}$ & Grow & Grow & Not grow & Grow & Grow & Not grow & Not grow \\
\hline G & Grow & Grow & Not grow & Grow & Grow & Grow & Not grow \\
\hline $\mathrm{H}$ & Grow & Grow & Not grow & Grow & Grow & Not grow & Not grow \\
\hline 1 & Not grow & Grow & Not grow & Grow & Grow & Not grow & Not grow \\
\hline J & Not grow & Grow & Grow & Grow & Grow & Grow & Not grow \\
\hline Q & Not grow & Not grow & Not grow & Not grow & Not grow & Not grow & Not grow \\
\hline K & Not grow & Grow & Not grow & Grow & Grow & Not grow & Not grow \\
\hline
\end{tabular}

for Salmonella bacteria in diets and suggests a low specificity of the culture Bacteriological versus techniques such as PCR. The absence of this Salmonella bacterium in the analyzes could also be explained by the sample size, because although 84 microbiological analyzes were performed on the 12 diets, an $n=12$ appears to be a low sample size to search for Salmonella and perhaps with a larger sample, or by analyzing several batches of each food, growth records of said microorganism could be obtained. Therefore, it is recommended that in future analyzes studies be carried out with a greater number of diets and that analyzes be obtained from different batches of each company, which would increase the possibility of finding more representative results. It is also recommended that, in future studies, PCR be considered as a more sensitive and specific technique, as has been done in other studies $[4,5]$.

The analyzes obtained are true and reliable, however, in order to give greater security to the results, it is recommended that in future studies analyzes of paired diets be carried out, where the same diet and the same batch are evaluated in 2 different laboratories, which would increase the reliability of the results. The BARF diets to which we had access in the city of Medellín do not have, for the most part, an adequate traceability system that guarantees the safety of their content. This is important because when an outbreak of any disease occurs in pets or even in their owners, or in the people who manufacture or handle BARF diets, only with a good monitoring and traceability system could the origin of the infection.

Furthermore, these diets could be a source of transmission of zoonotic bacterial and/or fungal diseases to humans, either by manipulation of diets, cross-contamination of food or by contact with contaminated animals that consume them. The results obtained provide an insight into future more in-depth studies, with more sensitive techniques and with more relevant samples that allow us to define more clearly the great risk to public health of the BARF and RMBD diets $[2,4,5]$. The people most susceptible to infection by contact with BARF diets are children, the elderly and immune compromised people, who could easily suffer serious complications when coming into contact with BARF-RMBD type foods, produced from raw meat [2].

The number of yeasts and fungi present in the BARF diets analyzed in this research work are alarming, not only because of the high levels of yeast, but also because they could generate mycotoxins that could seriously affect pets and the humans who handle them. The fungi, yeasts and bacteria present in the BARF diets, produced based on raw meat, could spread and generate cross contamination towards the food of the pet owners, since these must be stored under freezing or refrigeration environmental conditions and generally the Pet owners store this type of diet together with their own food $[4-7,15,16]$.

It is important to communicate the data obtained in this study, since being the first of its kind to be published in Latin America, it should serve as a public health alert for both pet owners and veterinary doctors, who are responsible for recommending what diets, are ideal to offer pets. It is worrying that the majority of the companies analyzed do not have an ICA registry, which is regulatory for the production and marketing of pet food, and a direct correlation was also found between the probability of contamination with different pathogens evaluated and the registry ICA that the analyzed BARF diet companies did or did not have, since only 4 of the 12 companies analyzed had such a register and here it is identified that, although it does not guarantee the safety and sanitary quality of the food, if there is a direct correlation between a lower probability of contamination for diets that have the ICA record compared to those that do not.

Animal health regulations should be stricter with regulations for the commercialization of BARF diets, and product labels should include warnings and instructions on the handling and use of these types of products, as has been suggested in other studies [16]. In addition, it would be important to review the current regulations on bacterial permissibility of pet food, since it has been in force for more than 20 years and could be outdated based on international parameters such as those of the FDA. BARF or RMBD-type raw meat-based pet foods have, in addition to meat as the main source of protein, other animal and vegetable by-products, dairy products, oils, meat meals, eggs, among others, and all are very prone to quick and easy decomposition unlike commercial concentrates for pets, which makes them an excellent culture medium for different microorganisms with possible pathogenic potential that could poison pets $[5,7,11,14,15,17]$.

The growth of E. coli in $17 \%$ and of Clostridium perfringens in $8 \%$ of the RMBD diets made from raw meat analyzed in this study, shows the current problems to which people and pets who are in contact are exposed with these foods, which has also been seen in other studies 
[17]. Regarding the protein sources of the analyzed BARF diets, it can be established that chicken meat in the diets is the most contaminated with pathogenic microorganisms and chicken meat is established as the main source of protein used in BARF foods, present in $42 \%$ of the analyzed diets.

It is suggested to carry out more in-depth analyzes in Colombia in this regard, where pathogenic microorganisms can be classified, determine the presence of mycotoxins in the faeces, analyze animals that consume BARF-type diets and make stool cultures or PCR in search of microorganisms and parasites as in other studies $[5,15,18]$, it has been possible to determine, in diets based on raw meat, possible bacteria that are multi-resistant to antibiotics or with traces of drugs widely used in the animal production industry, such as ivermectins, growth, antibiotics among others $[13,19]$.

\section{Conflict of Interests}

The authors declare that they have no conflict of interest. This study was financed with their own resources.

\section{References}

1. Seneviratne M, Subasinghe DWD, Watson PJ (2016) A survey of pet feeding practices of dog owners visiting a veterinary practice in Colombo, Sri Lanka. Vet Med Sci 2: 106-116.

2. Hellgren J, Hästö LS, Wikström C, Fernström LL, Hansson I (2019) Occurrence of Salmonella, Campylobacter, Clostridium and Enterobacteriaceae in raw meat-based diets for dogs. Vet Rec 184: 442.

3. Parr J, Remillard R (2014) Handling Alternative Dietary Requests From Pet Owners. Vet Clin North Am Small Anim Pract 44: 667-688.

4. Fredriksson M, Heikkilä T, Pernu N, Kovanen S, Hielm A, et al. (2017) Raw Meat-Based Diets in Dogs and Cats. Vet Sci 4: 1-9.

5. Freek P, Gertie C, Mineur R, Franssen F, Giessen M, et al. (2018) Zoonotic Bacteria and Parasites Found in Raw Meat-Based Diets for Cats and Dogs. Vet Rec 182: 114-115.

6. Schlesinger $D$, Joffe $D$ (2011) Raw food diets in companion animals : A critical review. Can Vet J 52: 50-54.

7. Freeman LM, Chandler ML, Hamper BA, Weeth LP (2010) Current knowledge about the risks and benefits of raw meat-based diets for dogs and cats, Timely Topics in Nutrition of raw meat-based diets for dogs and cats. J Am Vet Med Ass 243: 1549-1558.
8. Dillitzer N, Becker N, Kienzle E (2011) Intake of Minerals, Trace Elements and Vitamins in Bone and Raw Food Rations in Adult Dogs. Br J Nutr 106 S53-S56.

9. Kazue N, Bezerra V, Stella J, Erilane C, Fireman R, et al. (2008) Salmonella spp., importante agente patogênico veiculado em alimentos. Ciênc saúde coletiva 13: 1675-1683.

10. Laflamme D, Izquierdo O, Eirmann L, Binder S (2014) Myths and Mis perceptions About Ingredients Used in Commercial Pet Foods Byproducts Corn Soy Wheat Grains Carbohydrates Preservatives. Vet Clin NA Small Anim Pract 44: 689-698.

11. Iennarella-Servantez C (2017) Evaluation of raw meat diets on macronutrient digestibility, fecal output, microbial presence, and general health status in domestic dogs. Thesis, lowa State University: Ames, lowa.

12. Jarvis N, Corliss A, Turki M, Hong S, Young M, et al. (2016) An overview of Salmonella thermal destruction during food processing and preparation. Food Control 68: 280-290.

13. Nilsson O (2015) Hygiene quality and presence of ESBL-producing Escherichia coli in raw food diets for dogs. Infect Ecol Epidemiol 5: 1-4.

14. Stavisky J, Radford D, Gaskell R, Dawson S, German A, et al. (2011) A Case-Control Study of Pathogen and Lifestyle Risk Factors for Diarrhoea in Dogs. Prev Vet Med 99: 185-192.

15. Schmidt M, Unterer S, Suchodolski J, Honneffer J, Guard B, et al. (2018) The fecal microbiome and metabolome differs between dogs fed Bones and Raw Food (BARF) diets and dogs fed commercial diets. PLoS One 13: 1-20.

16. Mehlenbacher S, Churchill J, Olsen K, Bender J (2012) Availability, Brands, Labelling and Salmonella Contamination of Raw Pet Food in the Minneapolis/St. Paul Area. Zoonoses Public Health. 59: 513-520.

17. Sarah M, Tara D, Grabenstein M, McConnell T, McGrath T, et al. (2014) Investigation of Listeria, Salmonella, and Toxigenic Escherichia coli in Various Pet Foods. Foodborne Pathog Dis 11: 706-709.

18. Selmi M, Stefanelli S, Bilei S, Tolli R, Bertolotti L, et al. (2011) Contaminated Commercial Dehydrated Food as Source of Multiple Salmonella Serotypes Outbreak in a Municipal Kennel in Tuscany. Vet Ital 47: 183-190.

19. Baede V, Broens, Mirlin P, Arjen J, Haitske G, et al. (2017) Raw pet food as a risk factor for shedding of extended-spectrum betalactamase-producing Enterobacteriaceae in household cats. PLoS One 12: 1-11. 